ARTICULO ORIGINAL

\title{
Caracterización epidemiológica del dengue en la Unidad de Terapia Intensiva Adultos. Hospital Central "Dr. Emilio Cubas" del Instituto de Previsión Social, Paraguay \\ Epidemiological characterization of dengue in the "Dr. Emilio Cubas" Adult Intensive Care Unit. Central Hospital of the Social Security Institute, Paraguay
}

\author{
*Fleitas Mendoza EE ${ }^{\mathrm{I}}$, Ortiz Ovelar $\mathrm{C}^{\mathrm{I}}$, Ferreira Gaona MI ${ }^{\mathrm{II}}$, Díaz Reissner CV ${ }^{\mathrm{II}}$ \\ IUnidad de Terapia Intensiva Adultos. Hospital Central "Dr. Emilio Cubas". Instituto de \\ Previsión Social. Paraguay

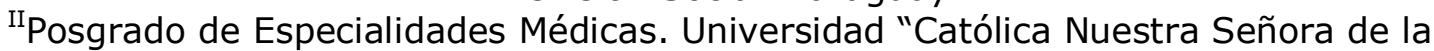 \\ Asunción". Paraguay
}

\section{RESUMEN}

El dengue es una infección viral sistémica, autolimitada y transmitida por mosquitos. En los últimos 50 años, a nivel mundial, aumentó la incidencia 30 veces, con circulación de los cuatro serotipos virales. Paraguay presenta condiciones socio-ambientales favorables para su transmisión, lo cual puede afectar la disponibilidad de recursos para el manejo de los casos graves. El objetivo del estudio fue determinar la frecuencia de dengue en la Unidad de Terapia Intensiva Adultos (UTIA) del Hospital Central del Instituto de Previsión Social, de junio del 2.011 a junio del 2.013. El estudio es observacional descriptivo, de corte transversal. De 1.991 pacientes admitidos en UTIA, en $103(5,2 \%)$ se confirmó dengue tanto por clínica como por hallazgos laboratoriales. El $72,7 \%$ era del sexo femenino, la mediana de edad fue de 47 años, y 53,4\% residía en Asunción. La mediana del tiempo hasta la primera consulta fue 2 días. El 87,4\% presentó fiebre; el 65,05\% sangrado y de estos el $52,2 \%$ requirió transfusión de hemoderivados. Las medianas de las puntuaciones APACHE II y SOFA fueron de 10 y 5, respectivamente. La mitad presentaba al menos una condición clínica coexistente. En promedio, la solicitud de la prueba NS1 fue a los $3 \pm 1$ días y la IgG/IgM a $6 \pm 2$ días. El $64,1 \%$ fue grave, complicándose el $73,8 \%$. La internación en cuidados intensivos fue en promedio 4 días. A pesar de la relativa baja frecuencia de esta enfermedad en la unidad, su relevancia radica en la gran morbimortalidad observada en estos pacientes.

Palabras clave: dengue grave, epidemiología, cuidados intensivos.

\section{ABSTRACT}

Dengue is a self-limiting systemic viral infection transmitted by mosquitoes. In the past 50 years, the incidence increased 30 times worldwide, with the circulation of the four viral serotypes. Paraguay has favourable socio-environmental conditions for its transmission, which may affect the availability of resources for the management of severe cases. The objective of this study was to determine the frequency of dengue in the Adult Intensive Care Unit (AICU) of the Central Hospital of the Social Security Institute from June 2011 to June 2013. It is a cross-sectional descriptive, observational study. Of 1,991 patients admitted to AICU, in $103(5.2 \%)$ dengue was confirmed by clinical and laboratory findings. Median age was 47 years, $72.7 \%$ was female and $53.4 \%$ resided in Asuncion. The median of time until the first consultation was 2 days. The most common clinical manifestation was fever $(87.4 \%), 65.1 \%$ presented bleeding and in this group $52.2 \%$ required hemoderivative transfusion. The medians of APACHE II and SOFA scores were 10

\footnotetext{
*Autor Correspondiente: Dr. Emilio Fleitas. Unidad de Terapia Intensiva Adultos. Hospital Central "Dr. Emilio Cubas". Instituto de Previsión Social. Paraguay

Email: emiliofleitas.md@gmail.com

Fecha de recepción: agosto 2014; Fecha de aceptación: enero 2015
} 
and 5, respectively. Half of the patients had at least one coexistent clinical condition. The NS1 test request was in average on $3 \pm 1$ days, and IgG/IgM on $6 \pm 2$ days; $64.1 \%$ of the cases were severe and $73.8 \%$ of these had complications. Median of days of hospitalization was 4 days. Despite the low frequency of dengue in the unit, its relevance is due to the high morbidity and mortality observed in these patients.

Keywords: severe dengue, epidemiology, intensive care.

\section{INTRODUCCIÓN}

El dengue es una infección viral sistémica, autolimitada, y transmitida por mosquitos de más rápida extensión en el mundo (1), con un amplio espectro de presentaciones clínicas, presentando evolución clínica y desenlace impredecibles (2).

El virus del dengue, un arbovirus del género Flavivirus, de la familia Togaviridae, presenta cuatro serotipos denominados DEN1, DEN2, DEN3 y DEN4: cada serotipo puede causar infección en una misma persona. La infección por un serotipo induce inmunidad de por vida contra el serotipo viral homólogo, pero solo tiene un efecto protector transitorio contra los demás tres serotipos $(3,4)$.

Según la Organización Mundial de la Salud (OMS), en los últimos 50 años, su incidencia se ha incrementado 30 veces en el mundo, con un aumento de la expansión geográfica a nuevos países y, en la presente década, de zonas urbanas a zonas rurales, convirtiéndose en una enfermedad de gran impacto epidemiológico, social y económico por lo que representa un preocupante problema para la salud pública regional y mundial $(2,5)$. La expansión global del dengue se debe a varios factores, entre ellas la amplia dispersión del Aedes aegypti, el eficaz mosquito vector, en gran parte del mundo tropical y subtropical, debido a una baja adherencia a los programas de control y a las condiciones socioambientales favorables a la expansión del vector que existen en estas zonas. El crecimiento poblacional, la urbanización no planificada, las pobres condiciones sanitarias y el deterioro en las infraestructuras de salud pública y su resultante limitado acceso a los cuidados de salud han contribuido, además, a la explosión de la enfermedad $(1,6)$.

A nivel mundial, en el año 2.010 más de 1,7 millones de casos de dengue fueron reportados, con 50.235 casos severos y 1.185 muertes. En Paraguay, el virus se introdujo en 1.988, con casos anuales que llevaron a convertirlo en endémico desde el 2.009. En el 2.010 fueron registrados 13.766 casos, en el 2.011 fueron 42.954, en el 2.012 se registraron 32.347 y en el 2.013 fueron 130.155, de acuerdo al boletín epidemiológico del Ministerio de Salud Pública y Bienestar Social (MSPBS). Al área metropolitana le correspondió el $60 \%$ de las notificaciones (7-9).

Las frecuentes epidemias a nivel mundial y los cambios en la epidemiología del dengue pusieron de manifiesto problemas con el uso de la clasificación de la OMS, vigente en las últimas décadas. La misma con sus criterios no lograba clasificar a muchos de los casos graves dentro de su grupo de riesgo, ni tampoco correlacionar todos los grupos con prácticas y decisiones clínicas tales como dónde y cuan intensivos deben los pacientes ser observados y tratados, por lo que se estableció una nueva clasificación $(2,10-12)$. La misma fue aprobada por el MSPBS en el año 2.012, y se encuentra disponible para su implementación y aplicación en el documento denominado "Guía de Manejo Clínico del Dengue"(13).

El desarrollo de cualquier signo de alarma en pacientes con diagnóstico de dengue indica la necesidad de hospitalización y observación estrecha, con el uso de líquidos por vía parenteral en pacientes con una ingesta oral inadecuada o un hematocrito en ascenso. Si la afección progresa al shock, la reanimación con líquidos para restaurar el volumen de plasma es imperativo, seguido de una fluidoterapia de mantenimiento para apoyar la circulación a un nivel suficiente para mantener la perfusión de los órganos nobles. Se deben utilizar soluciones cristaloides isotónicas, y las soluciones coloidales isotónicas 
deben reservarse para los pacientes con shock profundo o aquellos que no tienen una respuesta a la terapia inicial con cristaloides $(1,13-15)$.

La aparición de brotes recurrentes cada 3-5 años, con un número creciente de casos en el tiempo muestra la transición de un estado endémico-epidémico a un estado híperendémico en los últimos años. Este aumento dramático divulgado en el número de brotes demanda una acción urgente para evitar una mayor propagación de la enfermedad.

El objetivo de este estudio fue determinar la frecuencia y características de los pacientes con dengue en la Unidad de Terapia Intensiva Adultos (UTIA) del Hospital Central "Dr. Emilio Cubas" del Instituto de Previsión Social (HC-IPS), en el periodo comprendido de junio del 2.011 a junio del 2.013.

Se pretende alertar sobre el mencionado escenario epidemiológico estimulando iniciativas de control del vector por parte de la sociedad y las autoridades competentes así como exhortar a los colegas a la implementación y aplicación de las guías de manejo clínico del dengue, con el impacto que ello implica sobre las complicaciones y la mortalidad de la enfermedad.

\section{MATERIALES Y METODOS}

El diseño de estudio fue observacional descriptivo, temporalmente retrospectivo, de corte transversal. Fueron incluidos pacientes de ambos sexos, sin límite de edad, con diagnóstico clínico y laboratorial de dengue durante su internación en la unidad.

Las variables de interés fueron: a) Características demográficas: Edad, sexo, procedencia y fecha de internación; b) Características clínicas: Manifestación clínica inicial, motivo de consulta, tiempo de retraso hasta la primera consulta, manifestaciones clínicas más frecuentes, score APACHE II y SOFA de ingreso; c) Condiciones coexistentes; d) Hallazgos laboratoriales: Hemoglobina, hematocrito, plaquetas, NS1 y serología para dengue; e) Clasificación clínica: Dengue sin signos de alarma, con signos de alarma y dengue grave; f) Evolución clínica: complicaciones, días de internación y condición de egreso (alta médica u óbito).

Los datos fueron recolectados a partir de la revisión de fichas de pacientes internados en la unidad, que se encontraban registrados en la sala de estadística del mismo y analizados usando estadística descriptiva con el programa Excel ${ }^{\odot} 2013$ de Microsoft Corporation. Las variables cuantitativas se resumieron en medianas, medias y desviación estándar, mientras que las variables cualitativas se presentaron en frecuencias absolutas y porcentuales, en tablas.

El protocolo fue aprobado por el Comité de Ética en la Investigación del HC-IPS. En el desarrollo del estudio se respetaron los principios éticos en investigación en seres humanos de acuerdo a la declaración de Helsinki.

\section{RESULTADOS}

Un total de 1.991 pacientes ingresaron a la UTIA del HC-IPS durante el periodo de estudio. De ellos, 103 pacientes tuvieron diagnóstico confirmado de dengue tanto por clínica como por hallazgos laboratoriales (registrado en la historia clínica, sin aclaraciones del método utilizado), lo que representa una frecuencia de $5,17 \%$.

Del total de pacientes diagnosticados con dengue, el 78,6\% ingresó en el año 2.013 , el $19,4 \%$ en el 2.012 y $1,9 \%$ en el 2.011 , presentándose el mayor número de casos registrados en febrero del 2.013, con 19 casos.

La mediana de edad de los pacientes diagnosticados con dengue fue de 47 años, con una mínima de 16 años y máxima de 86 años; el 72,7\% fue de sexo femenino. Con respecto a la procedencia, el 53,4\% provenía de Asunción, el 37,86\% de Gran Asunción y $8,7 \%$ de otras regiones del país. El 24,3\% (25/103) del total de ingresos estaba constituido por embarazadas. 
La manifestación clínica inicial más frecuente fue la fiebre que marcó el inicio de la enfermedad en el $79,6 \%(82 / 103)$ de los pacientes. Como motivo de consulta más frecuente se encontró en primer lugar fiebre $(36,9 \%)$, dolor abdominal $(18,4 \%)$ y en tercer lugar alteración del sensorio (11,5\%) (Tabla 1).

Tabla 1. Motivos de consulta médica. Unidad de Terapia Intensiva Adultos. Hospital Central "Dr. Emilio Cubas" del Instituto de Previsión Social, Paraguay. Periodo: junio de 2011 a junio de 2013

\begin{tabular}{lrc}
\hline \multicolumn{1}{c}{ Manifestaciones clínicas } & Nro. & \% \\
\hline Fiebre & 38 & 36,9 \\
Dolor abdominal & 19 & 18,5 \\
Alteraciones del sensorio & 12 & 11,5 \\
Gingivorragia & 7 & 6,8 \\
Disnea & 6 & 5,8 \\
Vómitos & 6 & 5,8 \\
Epistaxis & 5 & 4,8 \\
Mialgias & 2 & 1,9 \\
Cefalea & 2 & 1,9 \\
Melena & 2 & 1,9 \\
Diarrea & 1 & 1,0 \\
Astenia & 1 & 1,0 \\
Genitorragia & 1 & 1,0 \\
Hematemesis & 1 & 1,0 \\
\hline
\end{tabular}

Con respecto al tiempo de retraso hasta la primera consulta médica, la mediana fue de 2 días, con tiempo de retraso mínimo de menos de un día y máximo de 12 días. Entre las manifestaciones clínicas más frecuentes, ya sea al principio, al momento de la consulta o durante su internación, se destacaron la fiebre, presente en $87,38 \%$ de todos los casos, seguida de mialgias en $60,2 \%$ (Tabla 2 ). Cabe mencionar que no se presentó ningún caso de tos, ni síndrome meníngeo y que algunos pacientes presentaron más de una manifestación.

En cuanto a las manifestaciones hemorrágicas, el 65,0\% (67/103) presentó algún tipo de sangrado, teniendo en cuenta que podrían presentar más de un tipo, de estos, presentaron gingivorragia el 35,8\%, (23/67) seguido de la epistaxis en el 23,9\% (16/67), genitorragia en el $19,4 \%(13 / 67)$ y otros sangrados tales como melena, hematemesis y hemoptisis en $14,9 \%(9 / 67), 13,4 \%$ (9/67) y 5,97\% (4/67), respectivamente. El 52,2\% (34/67) de los pacientes que presentó sangrados requirió transfusión de algún tipo de hemoderivado.

En cuanto al score APACHE II de los pacientes al ingreso, presentaron una mediana de 10 puntos, con una mínima de 0 y máxima de 34 puntos; y la mediana del score SOFA fue de 5 puntos, con una mínima de 0 y una máxima de 21 puntos.

En relación a las condiciones coexistentes, el 79,6\% (82/103) ingresó con algún tipo de condición coexistente relevante; $50,0 \%(41 / 82)$ de ellos con al menos una condición coexistente (41/82), $25,6 \%$ con 2 condiciones (21/82) y $24,4 \%$ con 3 o más condiciones coexistentes (20/82). Las más frecuentes fueron la hipertensión arterial en $58,5 \%$ de los pacientes $(48 / 82), 30,5 \%$ presentó diabetes mellitus tipo II $(25 / 82), 30,5 \%$ estaba cursando embarazo (25/82), 20,7\% presentó cardiopatías (17/82), insuficiencia renal crónica en $9,7 \%$ (8/82), asma/EPOC en 7,3\% (6/82), neoplasias en 4,9\% (4/82) y dislipidemia en $2,4 \%$ de los casos (2/82). Un 18,3\% presentó algún otro tipo de condición clínica relevante como ser artritis reumatoide, anticoagulación, úlcera gastroduodenal, 
vasculitis, miastenia gravis, polineuropatía motora, marcapasos cardiaco, síndrome de Down, cirrosis hepática, hipotiroidismo, enfermedad de Alzheimer, aneurisma de aorta.

Tabla 2. Manifestaciones clínicas. Unidad de Terapia Intensiva Adultos. Hospital Central "Dr. Emilio Cubas" del Instituto de Previsión Social, Paraguay. Periodo: junio de 2011 a junio de 2013

\begin{tabular}{lrr}
\hline \multicolumn{1}{c}{ Manifestaciones clínicas } & Nro. & \% \\
\hline Fiebre & 90 & 87,4 \\
Mialgias & 62 & 60,2 \\
Cefalea & 56 & 54,4 \\
Disnea & 54 & 52,4 \\
Artralgias & 41 & 39,8 \\
Vómitos & 40 & 38,8 \\
Dolor abdominal & 38 & 36,9 \\
Náuseas & 33 & 32,0 \\
Shock & 27 & 26,2 \\
Taquipnea & 25 & 24,3 \\
Gingivorragia & 24 & 23,3 \\
Sx. confusional & 23 & 22,3 \\
Petequias & 21 & 20,4 \\
Epistaxis & 16 & 15,5 \\
Genitorragia & 13 & 12,6 \\
Diarrea & 13 & 12,6 \\
Melena & 10 & 9,7 \\
Hematemesis & 9 & 8,7 \\
Exantema & 4 & 3,9 \\
Oligoanuria & 4 & 3,9 \\
Hemoptisis & 4 & 3,9 \\
Convulsiones & 4 & 3,9 \\
Dolor retroocular & 3 & 2,9 \\
Ictericia & 3 & 2,9 \\
Prurito & 2 & 1,9 \\
Inyección conjuntival & 1 & 1,0 \\
\hline
\end{tabular}

Las cifras de hemoglobina y hematocrito promedio al ingreso fueron $11,4 \pm 2,7 \mathrm{mg} / \mathrm{dL}$ y $34,6 \pm 7,9 \%$, respectivamente; con valores mínimo y máximo de $4,20 \mathrm{mg} / \mathrm{dL}$ y $19 \mathrm{mg} / \mathrm{dL}$ para la hemoglobina; y de $12,9 \%$ y $55,0 \%$ para el hematocrito. No hubo registro uniforme de la evolución de estas variables en el periodo de permanencia en la UTIA.

La mediana de recuento de plaquetas al ingreso fue de 58.000 plaquetas $/ \mathrm{mm}^{3}$, con valores mínimos y máximos de 12.000 y 300.000 plaquetas $/ \mathrm{mm}^{3}$, respectivamente.

En promedio, el test de antigenemia NS1 se solicitó a partir del día $3 \pm 1$ de enfermedad, siendo esta la prueba diagnóstica confirmatoria en un $77,7 \%$ de los pacientes. Las pruebas serológicas IgG e IgM para dengue fueron solicitadas a partir del día $6 \pm 2$ de enfermedad, llegándose al diagnóstico de dengue mediante esta prueba en el $22,3 \%$ de los pacientes. 
Un $64,1 \%$ de los casos ingresados a UTIA durante el periodo de estudio correspondió a dengue grave (66 casos), el 34,9\% correspondió a dengue con signos de alarma (36/103) y el $0,97 \%$ a dengue sin signos de alarma (1/103). La mayor parte de los casos graves ingresó en el 2.013 (Tabla 3).

Tabla 3. Clasificación de gravedad del dengue por año. Unidad de Terapia Intensiva Adultos. Hospital Central "Dr. Emilio Cubas" del Instituto de Previsión Social, Paraguay. Periodo: junio de 2011 a junio de 2013

\begin{tabular}{lrrrrrrrr}
\hline \multicolumn{1}{c}{ Dengue } & \multicolumn{2}{c}{$\mathbf{2 0 1 1}$} & \multicolumn{2}{c}{$\mathbf{2 0 1 2}$} & \multicolumn{2}{c}{$\mathbf{2 0 1 3}$} & \multicolumn{2}{c}{ Total } \\
& Nro. & \% & Nro. & \multicolumn{1}{c}{$\%$} & Nro. & \multicolumn{1}{c}{$\%$} & Nro. & \multicolumn{1}{c}{$\%$} \\
\hline Grave & 1 & 1,0 & 13 & 12,6 & 52 & 50,5 & 66 & 64,1 \\
Con signos de alarma & 1 & 1,0 & 7 & 6,8 & 28 & 27,2 & 36 & 35,0 \\
Sin signos de alarma & -- & -- & -- & -- & 1 & 1,0 & 1 & 1,0 \\
Total & 2 & 1,9 & 20 & 19,4 & 81 & 78,6 & 103 & 100,00 \\
\hline
\end{tabular}

Durante la evolución clínica, 73,8\% presentó algún tipo de complicación siendo las más frecuentes shock, 56,6\%; falla renal aguda o crónica agudizada, 34,2\%; afectación hepática desde hepatitis por dengue hasta falla hepática aguda, 32,9\%; complicaciones en el aparato digestivo (hemorragia digestiva, laparotomía en blanco, peritonitis apendicular), 30,3\%; neumonía asociada a la ventilación, 21,1\%; complicaciones cardíacas, $17,1 \%$ (incluyendo un caso de miocarditis por dengue); y $14,5 \%$ con complicaciones neurológicas (incluyendo 3 casos de encefalitis por dengue y 8 casos de accidente cerebrovascular tanto isquémico como hemorrágico).

La mediana de días de internación de los pacientes en el servicio fue de 4 días, con una mínima de 1 día y una máxima de 52 días.

En cuanto a la condición de egreso, 32,0\% de los pacientes falleció durante su internación, $97,0 \%$ de estos fueron casos de dengue grave (32 de los 33 óbitos reportados); siendo la tasa de mortalidad por dengue en UTIA de 16,5 muertes por cada 1.000 pacientes del servicio y la tasa de letalidad por dengue $22,3 \%$.

\section{DISCUSION}

En el presente trabajo de investigación, en UTIA en el periodo de junio del 2.011 a junio del 2.013, fue confirmado con dengue el 5,17\% de los pacientes ingresados en dicho periodo mientras que a nivel país se reportó una frecuencia de 2,64\% de casos en el 2.013 (16).

La proporción de casos de dengue fue mayor en el sexo femenino, con una relación hombre/mujer de 1:3, lo que coincide con los reportes regionales. Existen dos posibles hipótesis que podrían explicar estos datos: el hecho de que las mujeres accedan a los servicios de salud más que los hombres y por lo tanto el dengue sea notificado con mayor frecuencia entre ellas, y que las mujeres pasan más tiempo dentro de la residencia, el hábitat preferido del mosquito Aedes aegypti, por lo que están más expuestas a la enfermedad (17).

Poco más de la mitad de los casos provino del área metropolitana de Asunción $(53,40 \%)$ y una pequeña proporción de otras regiones del país $(8,74 \%)$, lo que podría deberse al carácter epidémico del dengue en el área metropolitana durante el periodo de estudio, que probablemente ocasionaba saturación del sistema de salud en el departamento Central. Además, hay que destacar los esfuerzos del MSPBS por disponer la implementación y aplicación de las guías de manejo de dengue, que facilitan las decisiones clínicas tales como dónde y cuán intensivos deben ser los pacientes observados y tratados, evitándose así las derivaciones innecesarias hacia la capital del país $(8,13,16,18)$. 
La población adulta fue la más afectada, siendo la mediana de edad de 47 años. Este grupo comprende a la mayoría de la población económicamente activa, que mayormente corresponde a los asegurados de la previsional, que circula con más frecuencia tanto dentro como fuera del país, y por lo tanto están sujetos a la exposición a un mayor número de focos del mosquito vector.

Se encontró un franco predominio de casos en el año 2.013 , coincidente con la que se describe como la peor epidemia de dengue en nuestro país $(16,19-21)$. Tres cuartas partes de los ingresos se dieron en dicho año contra una quinta parte y menos en los años 2.012 y 2.011 , respectivamente.

Como motivo de consulta más frecuente se encontró en primer lugar fiebre, principal síntoma de dengue reconocido por los pacientes paraguayos encuestados por la Dirección General de Promoción en Salud del MSPBS en el estudio realizado en el año 2.013 (22).

Las manifestaciones clínicas más frecuentes fueron la fiebre, cefalea, mialgias y artralgias, que se describen como las más frecuentes en la literatura, así como también, en varios estudios regionales y mundiales $(1,2,6,13,23-26)$.

Las manifestaciones hemorrágicas se presentaron en el $59,22 \%$ de los pacientes estudiados, frecuencia aproximada a la reportada por Taboada et al., en población internada en el Instituto de Medicina Tropical, encontrándose desde gingivorragias autolimitadas hasta sangrados digestivos masivos, hemorragias intracraneales 0 metrorragias severas. Cabe destacar que casi dos tercios de los pacientes con manifestaciones hemorrágicas requirió transfusión de algún tipo de hemoderivados (26).

Factores específicos del huésped pueden determinar la severidad de la enfermedad y entre ellos se describen las enfermedades crónicas como la diabetes mellitus, el asma, hipertensión arterial, insuficiencia renal crónica, neoplasias, etc. En total, 79,61\% de los pacientes ingresados con dengue presentó algún tipo de comorbilidad, la mitad de ellos con al menos una condición coexistente, la cuarta parte con 2 condiciones y el resto con 3 o más condiciones coexistentes, lo que probablemente los expuso a desarrollar formas más graves de la enfermedad (2).

Una condición relevante es el embarazo, dado que algunas de las manifestaciones del dengue pueden aumentar los riesgos del embarazo o confundirse con complicaciones del mismo. Las principales complicaciones del dengue mencionadas en la literatura en este contexto son: parto pretérmino, hemorragias durante la cesárea, así como una mayor incidencia de muerte fetal. También se ha señalado un incremento en el riesgo de abortos $(27,28)$. Cabe destacar, que del total de los ingresos en UTIA, el $24,27 \%$ estaba cursando embarazo, en los que la evolución del dengue suponía un riesgo para su vida y la del producto (29).

Respecto a los métodos diagnósticos confirmatorios, el test de antigenemia NS1 y la serología IgG/IgM para dengue fueron solicitados en los lapsos correspondientes ( $3 \pm 1$ días de evolución para el NS1, y $6 \pm 2$ días de evolución para las serologías), como dictan los protocolos nacionales e internacionales $(2,13)$.

Los casos de dengue grave corresponden a aquellos en donde existe escape importante de líquidos con shock, disnea, daño orgánico grave y/o sangrados importantes y según las guías de la OMS y las vigentes a nivel nacional deben ser manejados en una unidad de cuidados críticos $(1,2,13)$. Por tratarse de una población internada en cuidados intensivos, se observó una alta frecuencia (dos tercios) de casos graves.

Respecto a las complicaciones, la presente investigación arrojó un mayor reporte de complicaciones en comparación con las observadas unos años atrás (26), se desconoce si lo anterior obedece a un subregistro en el sistema. Se destaca el shock como complicación más frecuente en poco más de la mitad de los casos, seguido de falla renal, afectación hepática y complicaciones del aparato digestivo en 30,26\%. Complicaciones inusuales como encefalitis por dengue (3 casos), miocarditis por dengue (1 caso), hemorragia sub-aracnoidea ( 5 casos), hematomas intracraneales ( 2 casos) e insuficiencia hepática aguda ( 4 casos) también fueron reportadas. 
La mediana de días de internación de los pacientes en el servicio fue de 4 días, con un mínimo de 1 día y un máximo de 52 días. En el estudio realizado en el año 2.008 por Taboada et al., el promedio de días de internación fue de 1 (0-9 días) (26). Dicho estudio se realizó en pacientes internados en la sala de Clínica Médica del Instituto de Medicina Tropical. Es de destacar el mayor tiempo promedio de internación de los pacientes ingresados en UTIA en comparación con el tiempo de internación encontrado por el estudio de Taboada et al., lo cual resulta lógico al considerar que los pacientes que por su gravedad requieren internación en una unidad de cuidados críticos, demandan mayor cantidad de recursos humanos, económicos y de infraestructura que aquellos que no requieren internación en dicho nivel asistencial.

Respecto a las modificaciones en la clasificación y manejo de la enfermedad observadas en las guías de manejo de dengue actualizadas en el 2.009 por la OMS e implementadas por el MSPBS, son de gran utilidad al lograr prever mediante la valoración de los hallazgos clínicos y laboratoriales, que grupo de pacientes podrían estar evolucionando a formas graves de la enfermedad y así definir de manera clara y pragmática el manejo de casos respecto al tratamiento apropiado y oportuno, y el lugar donde este tratamiento debe ser instaurado (domicilio, sala de internación común o UTIA), optimizando así el resultado final.

Se debe considerar que este estudio fue efectuado en una unidad del sistema previsional paraguayo, debido a lo cual no es representativo de la población paraguaya.

En conclusión, se puede afirmar que la fiebre fue el motivo de consulta y la manifestación clínica inicial más frecuente entre estos pacientes. Las manifestaciones hemorrágicas fueron comunes y más de la mitad de ellas requirió transfusiones de hemoderivados. Una importante proporción de pacientes presentó comorbilidades, predominando la hipertensión arterial. Las pruebas NS1 y serologías IgG/IgM fueron solicitadas en los tiempos adecuados. Un importante número de pacientes presentó complicaciones, principalmente el shock. Dos tercios de los casos fueron dados de alta, siendo la tasa de letalidad por dengue de $22,33 \%$.

A pesar de encontrarse una frecuencia baja de dengue en UTIA en el periodo estudiado, ésta no deja de ser considerada una patología relevante, en vista a la gran morbimortalidad de los casos ingresados, en su mayoría de dengue grave.

Dado el hecho de que el dengue puede aumentar los riesgos de complicaciones durante el embarazo, se recomienda la prevención como la principal medida terapéutica, dado que no existe una medida terapéutica efectiva contra la infección por el virus del dengue. La misma premisa se recomienda para el grupo de pacientes con comorbilidades ya que se vio que presentan mayor predisposición a desarrollar formas graves de la enfermedad.

\section{REFERENCIAS BIBLIOGRÁFICAS}

1. Simmons CP, Farrar JJ, van Vinh Chau N, Wills B. Dengue. N Engl J Med. 2012; 366(15):142332.

2. World Health Organization, Special Programme for Research and Training in Tropical Diseases. Dengue: Guidelines for diagnosis, treatment, prevention, and control. Geneva: World Health Organization; 2009.

3. Chokephaibulkit K, Perng GC. Challenges for the formulation of a universal vaccine against: Dengue. Exp Biol Med. 2013; 238(5):566-78.

4. Blok J. Genetic relationships of the dengue virus serotypes. J Gen Virol. 1985; 66(6):1323-5.

5. OPS, OMS. Estrategia de gestión integrada de prevención y control de dengue para los Estados Partes y Asociados del MERCOSUR: IGI - dengue MERCOSUR [Internet]. Asunción: OPS, OMS; 2007 [citado 20 dic 2013]. Disponible en: http://new.paho.org/hq/dmdocuments/2010/EGIDENGUE\%20ESTADOS\%20PARTES\%20Y\%20ASOCIADOS\%20DEL\%20MERCOSUR.pdf

6. San Martín JL, Brathwaite O, Zambrano B, Solórzano JO, Bouckenooghe A, Dayan GH, et al. The epidemiology of dengue in the Americas over the last three decades: A worrisome reality. Am J Trop Med Hyg. 2010; 82(1):128-35.

7. Brathwaite Dick O, San Martín JL, Montoya RH, del Diego J, Zambrano B, Dayan GH. The history of dengue outbreaks in the Americas. Am J Trop Med Hyg. 2012; 87(4):584-93. 
8. Ministerio de Salud Pública y Bienestar Social, Dirección General de Vigilancia de la salud. Dengue informaciones útiles. Boletín Epidemiológico [Internet]. 2012[citado 20 dic 2013]; (50): 11. Disponible en: http://vigisalud.gov.py/images/documentos/boletines/2012.

12.28\%20boletin\%20epidemiologico.pdf

9. Cabello A, Von Horoch M, Ojeda A, Mendieta O, Lima P, Allende I, et al. Plan de acción para la prevención y el control del dengue: Set 2013 - set 2014 [Internet]. Asunción: DGVS. [citado 20 dic 2013]. Disponible en: http://www.mspbs.gov.py/rs-12/wp-content/uploads/2013/12/Plan-deAcci\%C3\%B3n-para-la-Prevenci\%C3\%B3n.pdf

10. Guha-Sapir D, Schimmer B. Dengue fever: new paradigms for a changing epidemiology. Emerg Themes Epidemiol. 2005;2(1):1.

11. Deen JL, Harris E, Wills B, Balmaseda A, Hammond SN, Rocha C, et al. The WHO dengue classification and case definitions: Time for a reassessment. Lancet. 2006; 368(9530):170-3.

12. Bandyopadhyay S, Lum LCS, Kroeger A. Classifying dengue: A review of the difficulties in using the WHO case classification for dengue haemorrhagic fever. Trop Med Int Health. 2006; 11(8):1238-55.

13. Ministerio de Salud Pública y Bienestar Social. Dengue: Guía de Manejo Clínico [Internet]. Asunción: MSPBS; 2012 [citado 20 dic 2013]. Disponible en:

http://www.mspbs.gov.py/documentacion/Dengue_guia_2012.pdf

14. Hung NT. Fluid management for dengue in children. Paediatr Int Child Heal. 2012; 32(s1):3942.

15. Wills BA, Dung NM, Loan HT, Tam DTH, Thuy TTN, Minh LTT, et al. Comparison of three fluid solutions for resuscitation in dengue shock syndrome. N Engl J Med. 2005; 353(9):877-89.

16. Ministerio de Salud Pública y Bienestar Social, Dirección General de Vigilancia de la salud. Enfermedades de notificación obligatoria: Dengue. Boletín Epidemiológico [Internet]. 2013[citado 20 dic 2013]; (47): 2-9. Disponible en: http://vigisalud.gov.py/images/documentos/boletines/

2013.12.16\%20boletin\%20epidemiologico.pdf

17. Cardoso IM, Souza Areias Cabidelle A, Castro e Leao Borges P, Ferreira Lang C, Calenti FG, Oliveira Nogueira L, et al. Dengue: Clinical forms and risk groups in a high incidence city in the southeastern region of Brazil. Rev Soc Bras Med Trop. 2011; 44(4):430-5.

18. Ministerio de Salud Pública y Bienestar Social, Dirección General de Vigilancia de la salud. Dengue: Datos del año 2011 hasta la SE 52. Boletín Epidemiológico [Internet]. 2011[citado 20 dic 2013]; (49): 3-6. Disponible en: http://vigisalud.gov.py/images/documentos/boletines/

2011.12.30\%20boletin\%20epidemiologico.pdf

19. Organización Panamericana de la salud. Salud: Alarma por la epidemia de dengue en América Latina, pero La Habana no informa de casos. Diario de Cuba [Internet]. 20 nov 2013 [citado 20 dic 2013]. Disponible en: http://www.diariodecuba.com/cuba/1384904898_5995.html

20. Arbo A. La epidemia más grave. ABC Color: Nacionales [Internet]. 29 abr 2013 [citado 20 dic 2013]. Disponible en: http://www.abc.com.py/nacionales/la-epidemia-mas-fuerte-y-grave566484.html

21. Barán MT, Agüero C, Ortega S, Basualdo O, Afara J. Paraguay: 242 muertos por dengue, el peor registro de su historia. Infobae América [Internet]. 17 nov 2013. [citado 20 dic 2013]. Disponible en:http://www.infobae.com/2013/11/17/1524322-paraguay-242-muertos-dengue-elpeor-registro-su-historia

22. Ministerio de Salud Pública y Bienestar Social. Resultados de investigación basada en encuesta en hogares de la XI Región Sanitaria, Central y la XVIII Región Sanitaria, Capital, para identificar mensajes clave y medios necesarios para el fortalecimiento de la comunicación y la educación en la prevención y el control del Dengue [Internet]. Asunción: Dirección General de Promoción de la Salud; 2013 [citado20 dic 2013].Disponible en: http://www.mspbs.gov.py/promociondelasalud/wpcontent/uploads/2014/01/Encuesta-de-Dengue-2013-DGPS.pdf.

23. Farreras $P$, Rozman C. Medicina interna. 17a ed. Barcelona: Elsevier; 2012.

24. Kasper D, Braunwald E, Fauci A, Hauser S, Longo D, Jameson L. Harrison: Princípios de Medicina Interna. 18a ed. New York: McGraw-Hill Interamericans; 2012.

25. Vázquez C. Características virológicas y serológicas de pacientes con dengue grave y fallecidos por dengue durante la epidemia del año 2011 en Paraguay. Rev Inst Med Trop. 2012; 7(1): 8-18. 26. Taboada A, Insfrán I, Benítez G, Kunzle C. Nuestra experiencia en la última epidemia de dengue. Rev Inst Med Trop. 2008; 3(1):21-8. 
27. Carroll ID, Toovey S, Van Gompel A. Dengue fever and pregnancy: A review and comment. Travel Med Infect Dis. 2007; 5(3):183-8.

28. Carles G, Talarmin A, Peneau C, Bertsch M. Dengue fever and pregnancy. A study of 38 cases in french Guiana. J Gynécologie Obstétrique Biol Reprod. 2000; 29(8):758-62.

29. Arbo A, Basualdo W, Bogado V, Iramain R, Lovera D, Pavlicih V. Manejo de casos de dengue durante epidemias. Pediatr (Asunción). 2011 abr; 38(1):57-62. 